\title{
PREDICTIVE OPTIMIZING REFERENCE GOVERNOR FOR CONSTRAINED 2 DOF's ROBOT WITH ABRUPT SET-POINT TRAJECTORIES
}

\author{
Ali Benniran \\ Faculty of Engineering, Sabratha University \\ benniran1@yahoo.com, alemqasm@gmail.com
}

\begin{abstract}
This paper discusses application of the predictive optimizing reference governor (multi-layer control strategy) to a process operates under basic feedback (unconstrained) controllers. The process is two degrees of freedom IMI robot equipped with PD-controllers. The PD-controllers have been considered as a direct (basic) control layer in the inner feedback loop of the hierarchical control scheme. The direct controllers receive their reference trajectory values (optimum set-point values) from a nonlinear constrained predictive optimizing governor (outer loop). The system objective is to fulfil both constraints and position tracking performance. The IMI robot is direct driven (DDA), has nonlinear dynamics of high coupling. These dynamics are linearized, at each sampling time, about the generated optimum values from application of Taylor's series method. The Matlap code simulation results prove the advantageous of the applied technique.
\end{abstract}

Keywords: multi-layer, reference governor, predictive control, industrial robot, multivariable control, non-linear control system

\section{Introduction}

Constraints are inherently characterizing almost all practical control systems, appearing most commonly as actuator bounds on control variables. However, other constraints on inputs, outputs and/or states also exist and are important. Violation of such constraints can drastically degrade the control schemes and in worst cases leads to instability (Qin\& Badgwell 2003, Kogiso \& Hirata 2003, Kogiso \& Hirata 2009). The underlying IMI robot, see figure 1, has build-in joint independent low level PDcontrollers, one controller for each joint (SISO control technique), which may be considered as Distributed Control System (DCS). The PD-controllers are capable of performing high position tracking accuracy (Spong et al 2006, Craig 2005). However, their main drawback is their disability to handle different constraints, in particular output constraints. Whereas in (Valle et al 2002), a constrained (input and output constraints) predictive control algorithm was successfully applied to a 2-DOF DDA robot. The main problem of the MPC technique is its computation burden 
particularly in case of applying a non-linear optimization problem. However this problem becomes not significant due to new computer capacities (Tatjewski 2007). It is quite nature that ideas of develop control systems utilize the advantage of different control strategies specially the optimality of MPC technique in form of multi-layer scheme applied in different areas. This approach decouples the problem of meeting constraints from obtaining a good local control design. The two layers may operate at different frequencies (Tatjewski et al 2009 ). Indeed, for example in (Tatjewski et al 2009, Bemporad 1998) the idea of multi-layer control (reference governor) strategy was discussed, where constraints, stability and tracking requirements are fulfilled by adding to a primal compensated nonlinear system

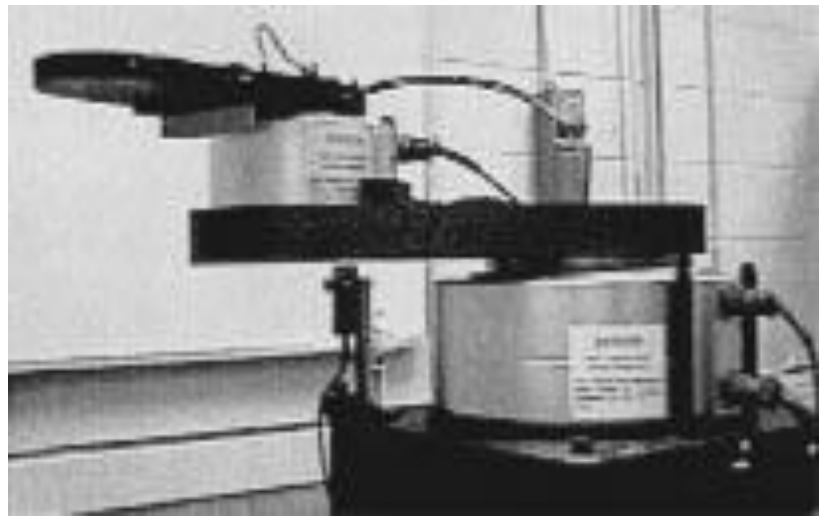

Figure 1: IMI robot.

Two different techniques were proposed. In (Tatjewski et al 2009) Two general approaches of model predictive control algorithms with economic steady-state optimization were investigated, namely approximate formulations of the target setpoint optimization and integration of MPC with economic optimization, whereas in (Bemporad 1998) the problem of satisfying point-wise-in-time input and/or state hard constraints in nonlinear control systems was proved. The approach is based on conceptual tools of predictive control and consists of adding to a primal compensated nonlinear system a reference governor. The resulting hybrid system proved to fulfil the constraints as well as stability and tracking requirements. Lastly but not least, in (Holaza et al 2017) the multi-layer technique is applied in a form of computationally tractable fashion, optimization-based reference governors for systems where multiple inner loops are closed by separate MPC techniques. The only outer loop does handle the constraints and hence bear the problem of optimize the references for the inner controllers.

In this paper, the multi-layer (reference governor) control is applied to IMI robot, as shown in scheme figure 2. The build-in PD-controllers form the direct control, which is the inner layer (subordinate feedback controller), whereas the MPC is the higher outer layer (supervisory). The direct control layer operates under the supervision of a 
higher control layer in a sense that the inner controllers receive optimized point-wise in time reference trajectories from the outer higher layer. The higher control layer takes the responsibility of generating the desirable reference trajectories optimum values, which at the same time prevents violation of the required constraints, for the direct controllers. The supervisory layer (governor) is designed as a MPC-NSL

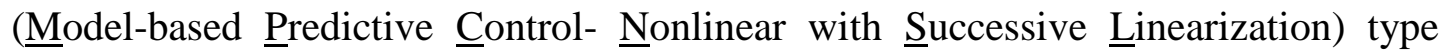
controller (Tatjewski 2007).

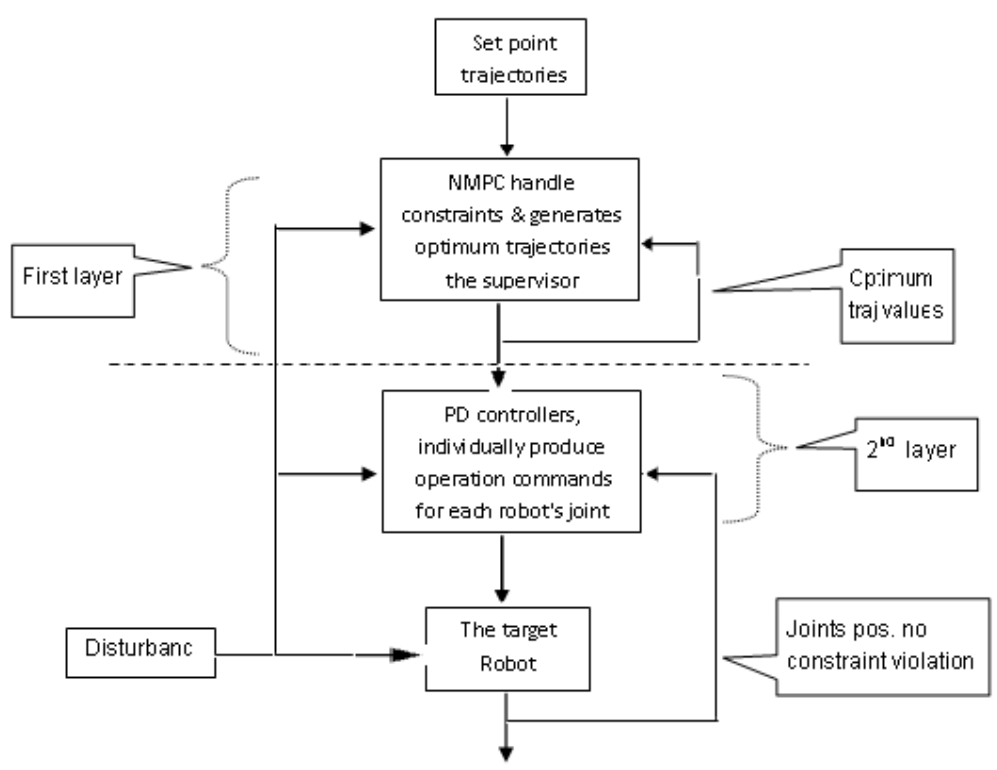

Figure 2: Multi-layer control structure.

The paper is organized as follows: Section 2, MPC-NSL algorithm and its optimization problem is described, in Section 3 presents process model dynamics and constraints, in Section 4, the applied control structure and terms calculation algorithm are presented. In Section 5 presents the simulation results and the paper ends with the conclusion in Section 6.

\section{MPC-NSL algorithm}

\section{Optimization problem}

The IMI robot has a nonlinear dynamics. The dynamical model is locally linearized at each sampling instant about the calculated optimum value of the previous sample. Consequently the used Nonlinear MPC (NMPC) algorithm is the MPC-NSL (ModelBased Predictive Control with Nonlinear with Successive Linearization) (Tatjewski 2007). At each consecutive sampling instant $k$ an optimal input control increment $\Delta \tau_{g}(k)$ and a set of future optimal control increments $\Delta U(k)$, over a control horizon $N_{u}$, is computed, where: 
$\Delta \tau_{g}(k)=\left[\Delta \tau_{g 1}(k), \Delta \tau_{g 2}(k)\right]$,

$\Delta U(k)=\left[\Delta \tau_{g}(k / k)^{T}, \Delta \tau_{g}(k+1 / k)^{T} \ldots \Delta \tau_{g}(k+N u-1 / k)^{T}\right]^{T}$

Leads to calculate: $\left.\tau_{g}(k / k)=\tau_{g}(k-1 / k)+\Delta \tau_{g}(k / k)\right]$,

$$
\left.\tau_{g}(k+1 / k)=\tau_{g}(k-1 / k)+\Delta \tau_{g}(k / k)+\Delta \tau_{g}(k+1 / k)\right], . . e t c
$$

$U(k)=\left[\tau_{g}(k / k)^{T}, \tau_{g}(k+1 / k)^{T}, \ldots, \tau_{g}\left(k+N_{u}-1 / k\right)^{T}\right]^{T}$

This optimal increments vector is a result of minimization of dynamic objective function containing in its first term the squares of the errors (deviation of the predicted outputs from the corresponding set-point trajectory points) vector:

$e(k+p / k)=q^{d}(k+p / k)-q^{p r d}(k+p / k) \ldots \forall p=1,2, \ldots \ldots . ., N_{p}$

and in its second term the squares of the future control increments vector $\Delta U(k)$, subjected to the plant constraints,

$$
\begin{aligned}
& \left.\begin{array}{l}
x(k)=A \cdot x(k-1)+B \cdot \tau_{g}(k-1) \\
q^{p r d}(k)=C \cdot x(k)
\end{array}\right\} \\
& \min _{\Delta U(k)}\left\{\left\|q^{d}(k)-q^{p r d}(k)\right\|_{\Psi}^{2}+\|\Delta U(k)\|_{\lambda}^{2}\right\} \\
& \text { s.to }\left\{\begin{array}{l}
-\Delta U_{\max } \leq \Delta U(k) \leq \Delta U_{\max } \\
U_{\text {min }}-U(k-1) \leq J \cdot \Delta U(k) \leq U_{\text {max }}-U(k-1) \\
q_{\text {min }}-q^{O}(k) \leq \Delta q(k) \leq q_{\max }-q^{o}(k)
\end{array}\right\}
\end{aligned}
$$

Where equation 3 represents the discrete state-space linearized model, in which $x$ is the state vector, $\mathrm{A}$ is the system matrix, $\mathrm{B}$ input matrix and $\mathrm{C}$ is the output matrix. Whereas equation 4 is the cost (objective) function, $\Delta U_{x}, U_{x} \in \mathfrak{R}^{n \cdot N_{u}}, U_{x}$ and $\Delta U_{x}$ is the maximum / minimum inputs and optimized increment vectors respectively, $N_{u}$ is the control interval and $J$ is a diagonal matrix of dimension $n . N_{p}$ its elements are identity matrices $n \times n . q^{d}, q_{x}, q^{o}, \Delta q \in \mathfrak{R}^{\text {outputvectorlength }}$ are the desired, maximum / minimum admissible predicted joint angles specified by the manufacturer (output constraints), free output joint angle and the forced output joint angle increments calculated over the prediction horizon $N_{p}$ respectively. In MPC strategies only the first set of increments, $\Delta \tau_{g}(k / k)$, corresponding to sampling instant $k$, is applied to the process: 
$\tau_{g}(k)=\left[\tau_{g 1}(k), \tau_{g 2}(k)\right]$,

$\left.\tau_{g_{i}}(k)=\left[\tau_{g_{i}}(k-1)+\Delta \tau_{g_{i}}(k \mid k)\right], \quad \forall i=1,2\right\}$

$\psi \geq 0$, and $\lambda \succ 0$ are diagonal weight matrices of dimensionality $n_{u} \cdot N_{p} \times n_{u} \cdot N_{p}$ and $n_{y} \cdot N_{u} \times n_{y} \cdot N_{u}$ respectively.

\section{Process dynamics and constraints}

The Euler-Lagrange equation of robot dynamics takes the following general form (Spong 2006, Graig 2005):

$$
\tau(k)=M(q(k)) \ddot{q}(k)+N(q(k), \dot{q}(k))+G(q(k))+F(q(k), \dot{q}(k))
$$

where; at instant time $k, M(q)$ is a $n \times n$ symmetric positive definite manipulator's inertia matrix, $N(q, \dot{q})$ is the $n \times 1$ vector of centrifugal and Coriolis terms, $F(q, \dot{q})$ is $n$ $\mathrm{x} 1$ vector representing viscous and Coulomb friction, $G(q)$ is the gravity vector, $\dot{q}$ and $\ddot{q}$ are the angular velocity and acceleration, and $n$ is the number of joints. Considering the IMI robot, values of the elements of these matrices and vectors are given by the compact form equation 7 :

$$
\left.\begin{array}{l}
M(q)=\left[\begin{array}{ll}
p_{1}+2 p_{3} \cos \left(q_{2}\right) & p_{2}+p_{3} \cos \left(q_{2}\right) \\
p_{2}+p_{3} \cos \left(q_{2}\right) & p_{2}
\end{array}\right] \\
N(q, \dot{q})=\left[\begin{array}{l}
-p_{3} \sin \left(q_{2}\right) \dot{q}_{2}-p_{3} \sin \left(q_{2}\right)\left(\dot{q}_{1}+\dot{q}_{2}\right. \\
p_{3} \sin \left(q_{2}\right) \dot{q}_{1}
\end{array}\right]\left[\begin{array}{l}
\dot{q}_{1} \\
\dot{q}_{2}
\end{array}\right] \\
F(q, \dot{q})=\left[\begin{array}{ll}
f_{d 1} & 0 \\
0 & f_{d 2}
\end{array}\right]\left[\begin{array}{l}
\dot{q}_{1} \\
\dot{q}_{2}
\end{array}\right]+\left[\begin{array}{ll}
f_{s 1} & 0 \\
0 & f_{s 2}
\end{array}\right]\left[\begin{array}{l}
\operatorname{sgn}\left(\dot{q}_{1}\right) \\
\operatorname{sign}\left(\dot{q}_{2}\right)
\end{array}\right]
\end{array}\right\}
$$

The nominal values of the manipulator parameters (the inertial parameters have been regrouped into the parameters, $p_{1}, p_{2}$ and $p_{3}$, the mass distribution is not given) are (Dixon et al 2000):

$p_{1}=3.473 \mathrm{kgm}^{2} ; p_{2}=0.193 \mathrm{kgm}^{2}$ and $p_{3}=0.242 \mathrm{kgm}^{2}$ whereas the friction constants as: $f_{d 1}=1.3 \mathrm{~N}, f_{d 2}=0.88 \mathrm{~N} ; f_{s 1}=1.519 \mathrm{Nm} / \mathrm{s}$, and $f_{s 2}=0.932 \mathrm{Nm} / \mathrm{s}$.

The gravity vector $G(q)$ equals to zero, because the robot has only horizontal motion.

\section{Constraints}

The joints' input torque limit (constraint) values are (Dixon et al 200):

$$
\tau_{\text {joint }(\max / \min )}= \pm[225.2,36.4] \mathrm{Nm}
$$


The output constraints are listed in table 1below, where subsequent columns contain: joints number, links length, twists angle, off-set and last column shows the maximum angles swept by joints movements.

Table 1. Shows Links parameters of the IMI Robot using modified D-H convention

\begin{tabular}{|c|c|c|c|c|c|}
\hline$i$ & $a_{i-1}$ & $\alpha_{i-1}$ & $d_{i}$ & $\vartheta_{i}$ & $\vartheta_{i}$ limits Degrees \\
\hline 1 & 0 & 0 & 0 & $\vartheta_{1}$ & $-25 \prec \vartheta_{1} \prec 40$ \\
\hline 2 & $L_{1}$ & 0 & $d_{2}$ & $\vartheta_{2}$ & $-170 \prec \vartheta_{2} \prec 170$ \\
\hline
\end{tabular}

where $L_{i}$ and $d_{i}$ are the length and off-set of link $i$ respectively.

\section{Desired trajectories}

Trajectories are defined in joint space coordinates. For simulation purpose, smooth trajectories of the $5^{\text {th }}$ order or higher polynomial, with respect to time, describing paths from initial $q_{\text {int }}$ position to the target position $q_{t r}$ in time $t_{f}$ with assumption of zero velocity and acceleration at start and end of the trajectories. Alternatively, abrupt motion trajectories (step trajectories) are used (Spong 2006). In this paper the later alternative is applied, because of high input changes are required, given by:

$q_{i}^{d}(t)=\left\{\begin{array}{l}1 \forall t>0, i=1,2 \\ 0 \forall t<0, i=1,2\end{array}\right.$,

where $q_{i}^{d}$ is the desired trajectory for joint $i$.

\section{Applied control scheme}

The applied control scheme consists of two layers, the first layer, reference governor, (also called constraint control and optimizer) and the second layer as shown in figure 2. The governor control layer has joints pre-determined desired trajectories described by equation 9 , whereas the second control layer receives the optimal joint set-point trajectories from the supervisory (governor) layer, as following:

\section{Governor}

It is a nonlinear device (computer program) occupying the higher layer in the hierarchical structure (see figure. 2). The governor's applied control law is the MPCNSL algorithm. The most important and distinctive role of this layer is to modify the reference (desired) trajectories supplied to the basic layer (closed-loop system, the direct controllers and plant) so as to enforce fulfilment of constraints and position tracking performance. 
At the end of each optimization operation, optimal values of the predicted joint positions, velocities and accelerations, denoted by, $q^{\text {prd }}, \dot{q}^{\text {prd }}, \ddot{q}^{\text {prd }}$ (are optimum quantities) are calculated, equations 3 , and sent to the PD controllers as desired values. The local control optimal input torques $\tau_{g}(k)$ is calculated at instant $k$, equation 4.

\section{Direct (Basic) controller}

The IMI's build-in PD-controllers will be considered as the direct controllers. Their roles are to generate the necessary manipulated variables vector (input to the IMI robot's joints) $\tau_{d}(k)$. The control input torque vector, equation 10 below, computed at sampling instant $k$, dropping the $\operatorname{argument}_{k}$ for space problem whenever clear, is calculated according to the formula $[4,5]$ :

$\tau_{d}(k)=M\left(\dot{q}^{o p t}+K_{p} e(k)+K_{v} \dot{e}(k)\right)+N(q, \dot{q})+F(\dot{q})$

$\ddot{q}_{d}=M^{-1}\left(q^{o p t}\right)\left[\tau_{d}-N\left(q^{o p t}, \dot{q}^{o p t}\right)-F\left(q^{o p t}, \dot{q}^{o p t}\right)\right]$

$\dot{q}_{d}(k)=\Delta T \ddot{q}_{d}(k)+\dot{q}_{d}(k-1)$

$q_{d}(k)=\Delta T^{\wedge} 2 \ddot{q}(k)+\Delta T \dot{q}_{d}(k-1)+q_{d}(k-1)$

where $K_{p} \in \mathfrak{R}^{n \times n}$ is the proportional gain diagonal matrix, $K_{d} \in \mathfrak{R}^{n \times n}$ is a diagonal matrix of derivative constants, $n=2 . e_{d} \in \mathfrak{R}^{n_{y}}$ is the angular position error vector $\left(e_{d}(k)\right.$ $=$ optimum joints position $q^{o p t}(k)$ - current joints position $q(k)$ calculated from equation 11), keep in mind that $q^{\text {opt }}(k)=q^{\text {prd }}(k)$ and, $\dot{e} \in \mathfrak{R}^{n_{y}}$ is the joint velocity error vector $(\dot{e}(k)$ =optimum joints velocity $\dot{q}^{\text {opt }}(k)$ - current joints velocity $\dot{q}(k)$ calculated from equation 11 and $\left.\dot{q}^{\text {opt }}(k)=\dot{q}^{\text {prd }}(k)\right), n_{y}=2$ is the number of outputs. $\ddot{q}^{\text {opt }}(k)$ is the second derivative of the optimal trajectory vector at instant $k_{k}$ generated in the first layer optimizer, equation 3. From equations 10 and 11 it is clear the coupling of the PD-controllers to the governor and the alignment of the direct layer to the constraints considered in the governor layer prevent from any constraints violation to occur.

\section{Simulation results}

Objectively, two models implemented in Matlap code are simulated:

1- Unconstrained and constrained systems operate under direct controllers alone,

2- Unconstrained and constrained systems involving the two layers technique.

\section{Simulation under bared unconstrained PD-controllers}

In this part the IMI robot is simulated under PD-controllers alone. The controllers are tuned for ultimate results of set-point tracking equation 9, minimum overshoot and 
short settling time. The result shows a quite satisfactory tracking (zero steady-state errors) but with intolerable inputs, high overshoots and long settling time, see figure 4.
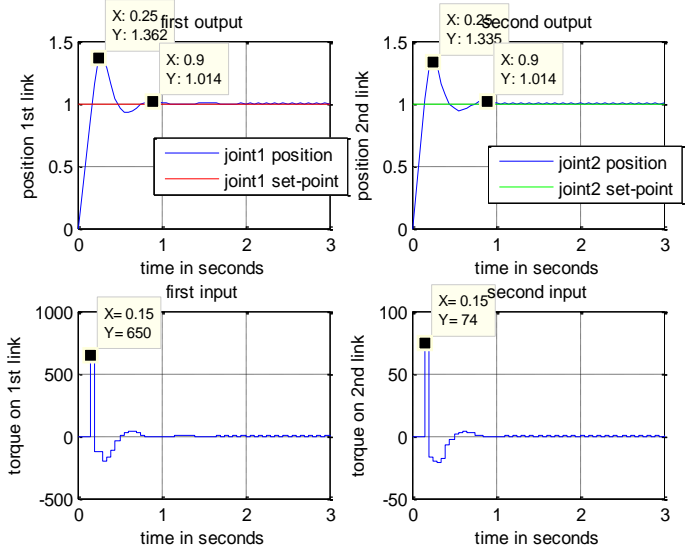

Figure 4: Simulation results under Un-constrained direct layer control only.

\section{Simulation under bared constrained PD-controllers}

Appling the same control tuned parameters that used in case 5.1, add the input constraints equation 8 . Adding of constraints certainly limits the required inputs, but do not significantly affect the overshoot or the settling time, see figure 5 .
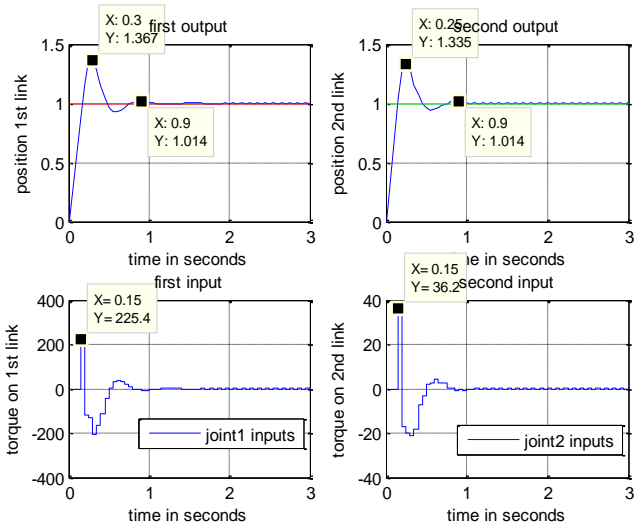

Figure 5: Simulation results under bared constrained PD-controllers

\section{Simulation involving Un-constrained governor}

In this part the process operates under the constructed multi-layered scheme, the outer layer reference governor supervisor the inner layer. The tuned direct layer control parameters used in case 5.1 are kept un-changed. The weights of the governor controller are tuned to achieve the goal; high tracking accuracy and short settling time with no overshoot. The governor's generated optimized output trajectories, shown in figure 6 . These optimum trajectories become the desired trajectories to the 
Un-constrained PD-controllers. It is worth to note that, although the constraints didn't considered, simulation results shown in figure 7 , the robot links position trajectories show high tracking with much less required input torques, compare with figure. 4; i.e. no input constraints violation. This is because of the predominant action of the governor, smoothing the desired trajectories.
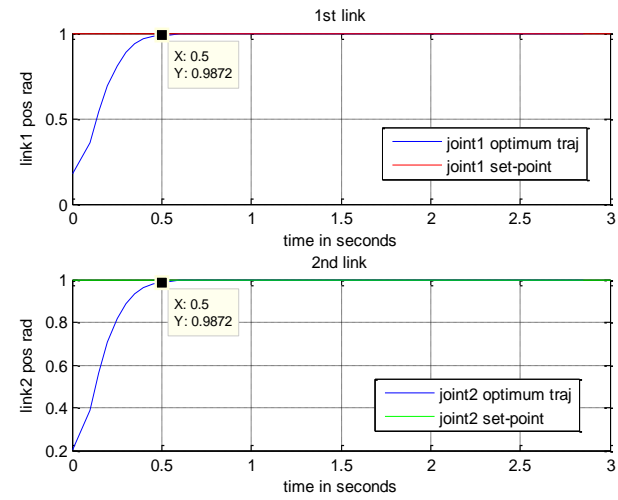

Figure 6: Optimum trajectories generated from the Un-constrained governor layer.
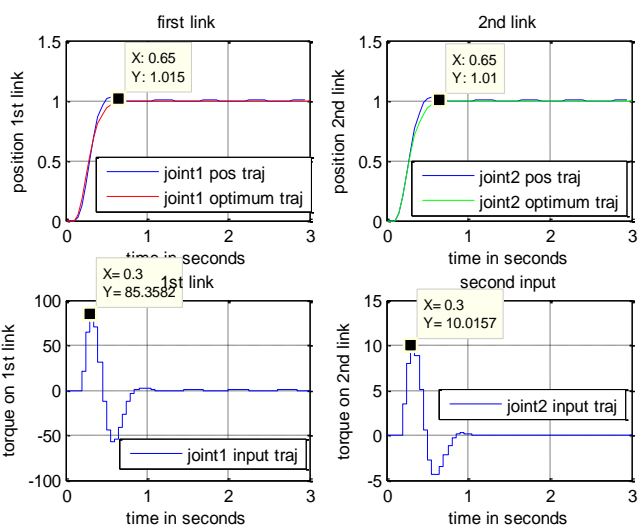

Figure 7: joint position and corresponding required input torques supervised by Unconstrained governor.

\section{Simulation involving constrained governor}

In this final part, simulation of the reference governor scheme (governor and PDcontrollers). The parameter values are kept unchanged, the case as of 5.2.1. The process constraints, equation 8 and table 1, are applied. Results are shown in figures 8 and 9 . Comparing figure 8 with figure 6 , it is noticed that the optimized trajectories values are little spread which means more smoothing of the trajectories. Also comparing figure 9 with figure 5, it is clear the high reduction in the required input torques and the great improvements in performance particularly, removing the overshoot and short settling time, zero steady-state error means; prevention from violation of any process's technical constraints. 

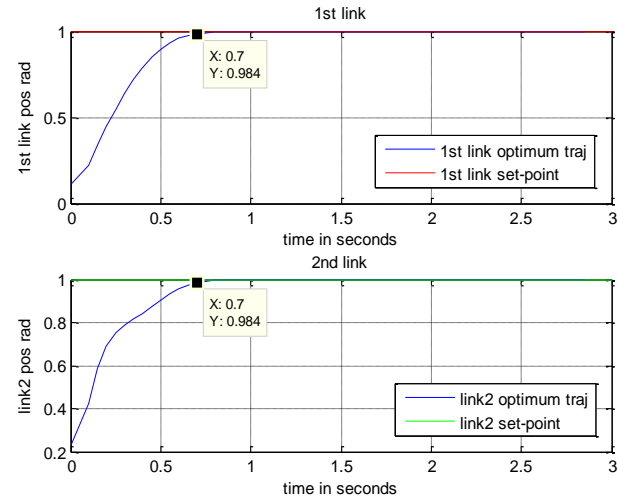

Figure 8: Optimum trajectories generated from the constrained governor layer.
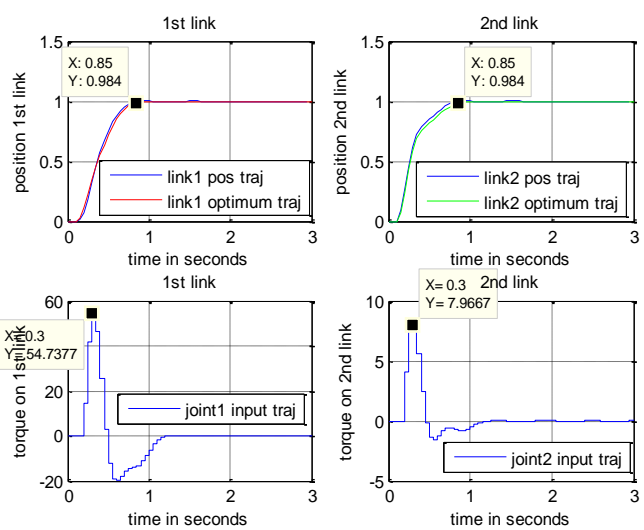

Figure 9: joint position and corresponding required input torques constrained governor.

\section{Conclusion}

In this paper the multi-layer control strategy (reference governor) was applied to nonlinear DDA robot for tracking reference trajectories at the same time considering process and operation technical constraints. The non-linear dynamics Robot directly controlled by PD classical control technique formed the target process. The process first simulated under unconstrained PD-controllers. Performance was satisfactory. The disadvantages are the required inputs torque are extremely high (violate the technical requirements) accompanying with long settling time and overshoot. The simulation is repeated where the process PD-controllers forms the inner loop for the predictive optimizing reference governor. The governor proposed in this paper is MPC-NSL strategy, developed for the purpose of optimizing trajectory and constraints handling. The developed system is simulated keeping the values of the PD-controller parameters un-changed. Performances of PD-controllers operate under the governor are improved, high reduction in inputs torque values and settling time were recorded. This is because of the outer loop (governor) smoothing the transients. 
The results show that, good path tracking performance, zero steady-state error, shorter settling time, no overshoot occurs, and no violation of the input and output constraints are achieved.

\section{Reference}

- S. Qin, T. Badgwell , 2003 “A survey of industrial model predictive control technology”, Control Engineering Practice 11, pp. 733-764.

- Kiminao Kogiso and Kenji Hirata, 2003 “A reference governor in a piecewise state affine function", proceedings of the $42^{\text {nd }}$ IEEE conference on decision and control Hawaii USA December 2003.

- Kiminao Kogiso and Kenji Hirata, 2009 "Reference governor for constrained systems with time-varying references", Robotics and Autonomous Systems 57, 289295.

- M. Spong, S. Hutchinson and M. Vidyasagar, Wiley 2006, "Robot Modelling and control”, pp. 213.

- John Craig, Pearson Prentice Hall, 2005, "Introduction to robotics, mechanics \& control”, pp. 201-216, 271-310.

- F. Valle, F. Tadeo and T. Alvarez, 2002 "Predictive control of robotic manipulators", Proceedings of the International conference on control applications, September 18-20, 2002, Glasgow UK.

- Piotr Tatjewski, Springer, 2007, “Advanced Control of Industrial Processes.

- Piotr Tatjewski Maciej Lawrynczuk and Piotr M. Marusak, 2009“Integrated predictive optimizer and constraint governor for processes with basic feedback control", European Control Conference ECC'2009, Budapest, 2009, 3359-3364.

- A. Bemporad, 1998, Reference governor for constrained nonlinear systems, IEEE Transactions on Automatic Control 43 (3), pp. 415-419.).

- Juraj Holaza et al, 2017, Slovak University of Technology in Bratislava Slovakia. "Solution Techniques for Multi-Layer MPC-Based Control Strategies", IFAC paper on-Line 50-1(2017) 15940-5.

- Warren E. Dixon et al, 2000 "Fault-Detection for Robot manipulator with Parametric Uncertainty: A Prediction-Error-Based Approach", IEEE Trans. On Robotics and Automation, vol. 16, no. 6, Dec. 2000. 